\title{
A fast Monte Carlo method for model-based prognostics based on stochastic calculus
}

\author{
M. Corbetta C. S. Kulkarni \\ SGT Inc., NASA Ames Research Center
}


Ingredients of model-based prognostic:

- state-space formulations

$$
\dot{x}=f_{\theta}(x, u, \omega)
$$

- Monte Carlo (MC) methods

$x^{(i)} \sim p(X)$ 
Ingredients of model-based prognostic:

- state-space formulations

$$
\begin{aligned}
& \dot{x}=f_{\theta}(x, u, \omega) \\
& x^{(i)} \sim p(X)
\end{aligned}
$$

- Monte Carlo (MC) methods

Some considerations:

- Monte Carlo methods are computationally expensive

- state-space formulations are differential equations with stochastic terms (SDE) 


\section{Contribution of this work}

Try to take advantage of stochastic calculus and SDE solutions to accelerate model-based prognostic using Monte Carlo simulations (?).

\section{Potential}

reducing computational time preserving (enhancing) the precision of estimations 
(1) Summary of model-based prediction

(2) Using stochastic calculus properties in model-based prediction

(3) Applications

- Case study 1: prognostic of electrolytic capacitors

- Case study 2: remaining time to discharge of Lithium-ion batteries

- Case study 3: fatigue damage prognosis of cracked structure

(4) Conclusions 
Summary of model-based prediction

$$
\begin{gathered}
\dot{x}=f_{\theta}(x, u, \omega) \\
\downarrow \\
x_{k}=x_{k-1}+f_{\theta}\left(x_{k-1}, u_{k-1}, \omega_{k-1}\right) \Delta t_{k}
\end{gathered}
$$




$$
\begin{gathered}
\dot{x}=f_{\boldsymbol{\theta}}(x, u, \omega) \\
\downarrow \\
x_{k}=x_{k-1}+f_{\boldsymbol{\theta}}\left(x_{k-1}, u_{k-1}, \omega_{k-1}\right) \Delta t_{k} \\
\downarrow \\
\text { Input: } x_{k}^{(i)} \sim p\left(x_{k}\right), \text { I }
\end{gathered}
$$$$
\text { Output: } x_{k+1}^{(i)} \sim p\left(X_{k+1}\right)
$$$$
\text { for each } x_{k}^{(i)} \sim p\left(X_{k}\right) \text { do }
$$$$
\text { for each } \tau \in\{1, \ldots, /\} \text { do }
$$$$
\omega_{k+\tau-1}^{(i)} \sim p\left(\Omega_{k+\tau-1}\right)
$$$$
x_{k+\tau}^{(i)}=x_{k+\tau-1}^{(i)}+f_{\theta}\left(x_{k+\tau-1}^{(i)}, u_{k+\tau-1}, \omega_{k+\tau-1}^{(i)}\right) \Delta t_{k+\tau-1}
$$$$
\text { end }
$$$$
\text { end }
$$ 


$$
\begin{gathered}
\dot{x}=f_{\boldsymbol{\theta}}(x, u, \omega) \\
\downarrow \\
x_{k}=x_{k-1}+f_{\boldsymbol{\theta}}\left(x_{k-1}, u_{k-1}, \omega_{k-1}\right) \Delta t_{k} \\
\downarrow \\
\text { Input: } x_{k}^{(i)} \sim p\left(x_{k}\right), \text { I }
\end{gathered}
$$$$
\text { Output: } x_{k+1}^{(i)} \sim p\left(X_{k+1}\right)
$$$$
\text { for each } x_{k}^{(i)} \sim p\left(X_{k}\right) \text { do }
$$$$
\text { for each } \tau \in\{1, \ldots, l\} \text { do }
$$$$
\omega_{k+\tau-1}^{(i)} \sim p\left(\Omega_{k+\tau-1}\right)
$$$$
x_{k+\tau}^{(i)}=x_{k+\tau-1}^{(i)}+f_{\theta}\left(x_{k+\tau-1}^{(i)}, u_{k+\tau-1}, \omega_{k+\tau-1}^{(i)}\right) \Delta t_{k+\tau-1}
$$$$
\text { end }
$$$$
\text { end }
$$ 
Using stochastic calculus properties in model-based prediction

State-space model utilized in prognostic (additive noise case)

$\dot{x}_{t}=f_{\theta}\left(x_{t}, u_{t}\right)+\omega_{t}$

$x_{k}=x_{k-1}+f_{\theta}\left(x_{k-1}, u_{k-1}\right) \Delta t_{k}+\omega_{k-1} \Delta t_{k}$ 
Using stochastic calculus properties in model-based prediction

State-space model utilized in prognostic (additive noise case)

$\dot{x}_{t}=f_{\theta}\left(x_{t}, u_{t}\right)+\omega_{t}$

$x_{k}=x_{k-1}+f_{\theta}\left(x_{k-1}, u_{k-1}\right) \Delta t_{k}+\omega_{k-1} \Delta t_{k}$

Typical SDE formulation

$\dot{X}_{t}=f_{\theta}\left(X_{t}, U_{t}\right)+\sigma\left(t, X_{t}\right) \xi_{t}$

$X_{t}=X_{0}+\int_{0}^{t} f_{\theta}\left(X_{s}, U_{s}\right) \mathrm{d} s+\int_{0}^{t} \sigma\left(s, X_{s}\right) \mathrm{d} B_{s}$

$X_{k}=X_{0}+\sum_{s=0}^{k-1} f_{\theta}\left(X_{s}, U_{s}\right) \Delta t_{s}+\sum_{s=0}^{k-1} \sigma\left(s, X_{s}\right) \Delta B_{s}$ 


\section{Using stochastic calculus properties in model-based prediction}

State-space model utilized in prognostic (additive noise case)

$\dot{x}_{t}=f_{\theta}\left(x_{t}, u_{t}\right)+\omega_{t}$

$x_{k}=x_{k-1}+f_{\theta}\left(x_{k-1}, u_{k-1}\right) \Delta t_{k}+\omega_{k-1} \Delta t_{k}$

Typical SDE formulation

$\dot{X}_{t}=f_{\theta}\left(X_{t}, U_{t}\right)+\sigma\left(t, X_{t}\right) \xi_{t}$

$X_{t}=X_{0}+\int_{0}^{t} f_{\theta}\left(X_{s}, U_{s}\right) \mathrm{d} s+\int_{0}^{t} \sigma\left(s, X_{s}\right) \mathrm{d} B_{s}$

$X_{k}=X_{0}+\sum_{s=0}^{k-1} f_{\theta}\left(X_{s}, U_{s}\right) \Delta t_{s}+\sum_{s=0}^{k-1} \sigma\left(s, X_{s}\right) \Delta B_{s}$

\section{Considerations}

under certain assumptions (e.g., $\sigma \neq \sigma\left(X_{t}\right)$ ), we can compute the SDE terms separately and we can find similarities between noise term and the diffusion term 


\section{Using stochastic calculus properties in model-based prediction}

State-space model utilized in prognostic (additive noise case)

$\dot{x}_{t}=f_{\theta}\left(x_{t}, u_{t}\right)+\omega_{t}$

$x_{k}=x_{k-1}+f_{\theta}\left(x_{k-1}, u_{k-1}\right) \Delta t_{k}+\omega_{k-1} \Delta t_{k}$

Typical SDE formulation

$\dot{X}_{t}=f_{\theta}\left(X_{t}, U_{t}\right)+\sigma\left(t, X_{t}\right) \xi_{t}$

$X_{t}=X_{0}+\int_{0}^{t} f_{\theta}\left(X_{s}, U_{s}\right) \mathrm{d} s+\int_{0}^{t} \sigma\left(s, X_{s}\right) \mathrm{d} B_{s}$

$X_{k}=X_{0}+\sum_{s=0}^{k-1} f_{\boldsymbol{\theta}}\left(X_{s}, U_{s}\right) \Delta t_{s}+\sum_{s=0}^{k-1} \sigma\left(s, X_{s}\right) \Delta B_{s}$

Considerations

under certain assumptions (e.g., $\sigma \neq \sigma\left(X_{t}\right)$ ), we can compute the SDE terms separately and we can find similarities between and the diffusion term 
Using stochastic calculus properties in model-based prediction

Let us consider $\sigma\left(t, X_{t}\right)=\sigma$ in the SDE:

$$
\int_{0}^{t} \sigma \mathrm{d} B \approx \sum_{s=0}^{k-1} \sigma \Delta B_{s}
$$


Let us consider $\sigma\left(t, X_{t}\right)=\sigma$ in the $\mathrm{SDE}$ :

$$
\int_{0}^{t} \sigma \mathrm{d} B \approx \sum_{s=0}^{k-1} \sigma \Delta B_{s}
$$

If we assume $\omega \sim \mathcal{N}(0, \sigma)$ in the state-space model:

$$
\omega_{k-1} \Delta t_{k}=\sigma z_{k-1} \Delta t_{k}=\sigma \Delta B_{k}
$$


Using stochastic calculus properties in model-based prediction

Some useful properties of Brownian motion $B$ :

- $\mathrm{d} B \sim \mathcal{N}(0, \mathrm{~d} t) \rightarrow \mathrm{d} B^{(i)}=\sqrt{\mathrm{d} t} z^{(i)}$

- $B_{t_{2}}-B_{t_{1}} \sim \mathcal{N}\left(0, t_{2}-t_{1}\right)$ 
Case study 1: prognostic of electrolytic capacitors ${ }^{1}$ :

$$
\begin{aligned}
\dot{C}_{l} & =\alpha C_{l}-\alpha \beta+\omega \\
C_{l}(t) & =e^{\alpha t}\left(-\beta+\beta e^{-\alpha t}+\int_{0}^{t} \sigma e^{-\alpha s} \mathrm{~d} B_{s}\right)
\end{aligned}
$$

${ }^{1}$ Celaya J, Kulkarni C, Biswas G, Saha S, Goebel K. A model-based prognostic methodology for electrolytic capacitors based on electrical overstress accelerated aging. Annual Conference of the PHM Society 2011; 25-29 Sept. 2011 
Case study 1: prognostic of electrolytic capacitors ${ }^{1}$ :

$$
\begin{gathered}
\dot{C}_{l}=\alpha C_{l}-\alpha \beta+\omega \\
C_{l}(t)=e^{\alpha t}\left(-\beta+\beta e^{-\alpha t}+\int_{0}^{t} \sigma e^{-\alpha s} \mathrm{~d} B_{s}\right) \\
\int_{0}^{t} \sigma e^{-\alpha s} \mathrm{~d} B_{s} \approx \sum_{s=0}^{k-1} \sigma e^{-\alpha t_{s}} \Delta B_{s}^{(i)}, \quad \forall i=1, \ldots, N
\end{gathered}
$$

${ }^{1}$ Celaya J, Kulkarni C, Biswas G, Saha S, Goebel K. A model-based prognostic methodology for electrolytic capacitors based on electrical overstress accelerated aging. Annual Conference of the PHM Society 2011; 25-29 Sept. 2011 
Case study 1: prognostic of electrolytic capacitors

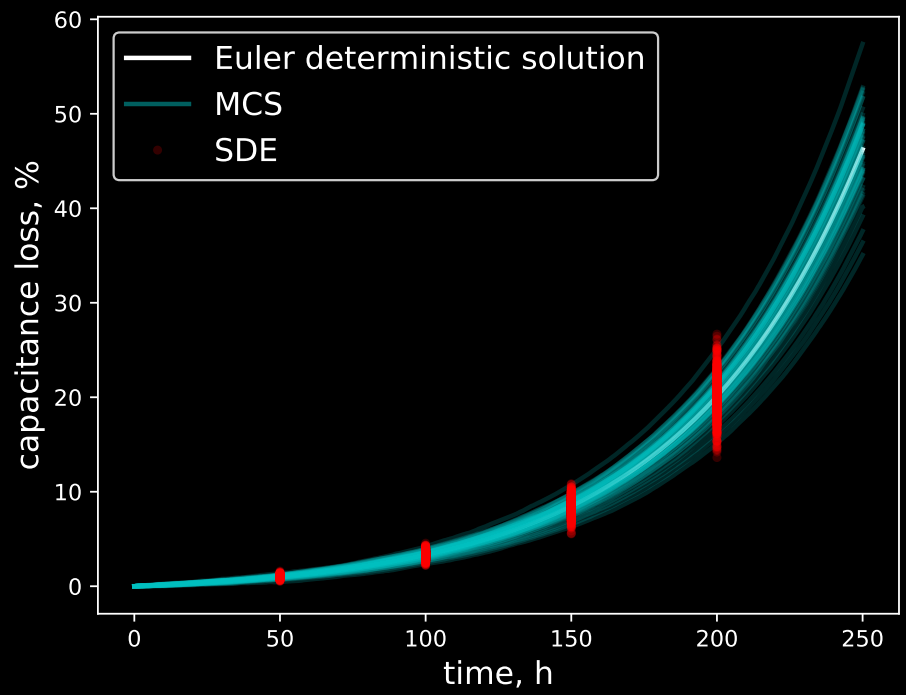


Case study 1: prognostic of electrolytic capacitors

Capacitance loss at $\mathrm{t}=50 \mathrm{~h}$

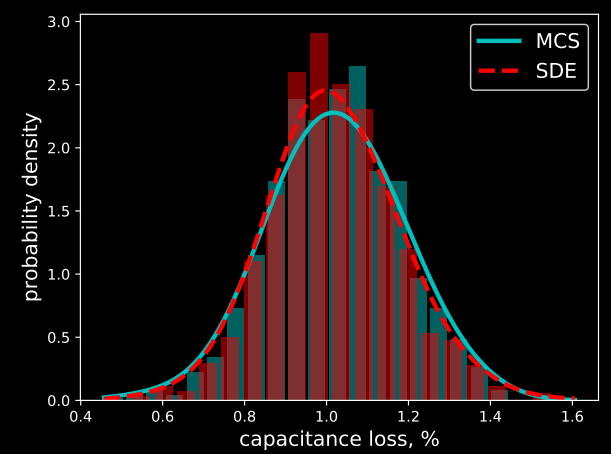

Capacitance loss at $\mathrm{t}=100 \mathrm{~h}$

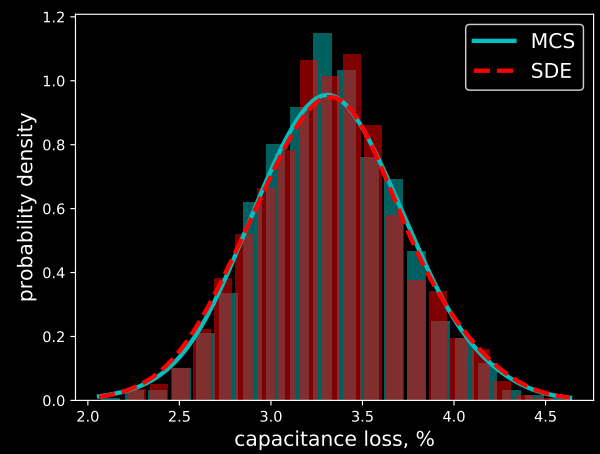


Case study 1: prognostic of electrolytic capacitors

Capacitance loss at $\mathrm{t}=150 \mathrm{~h}$

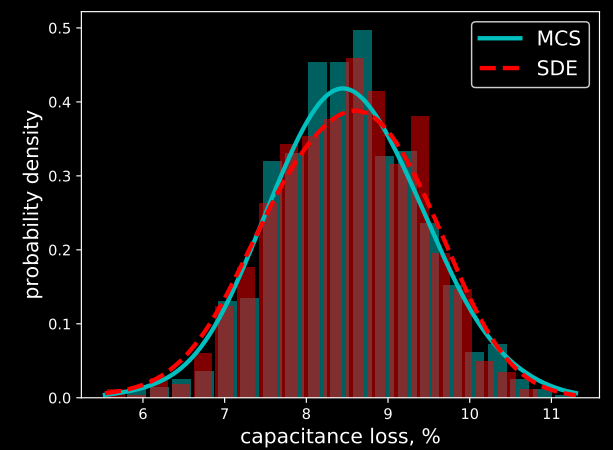

Capacitance loss at $\mathrm{t}=200 \mathrm{~h}$

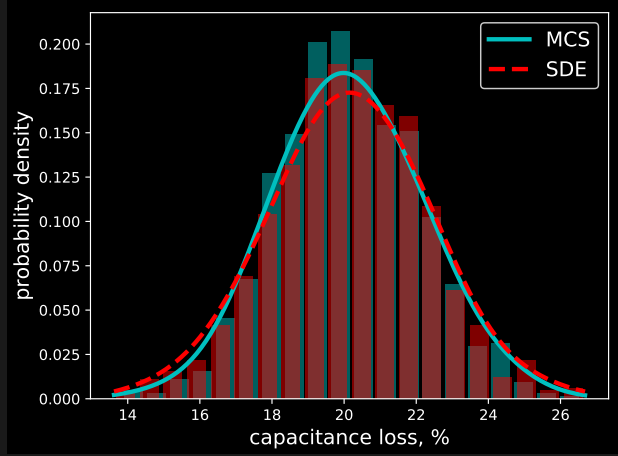


Case study 1: prognostic of electrolytic capacitors

\begin{tabular}{|c|c|c|c|c|c|}
\hline time $[\mathrm{h}]$ & $\mathrm{KL}\left(p_{\mathrm{MCS}} \| p_{\mathrm{SDE}}\right)$ & \multicolumn{2}{|c|}{$\begin{array}{l}\text { Hyp. test } @ \nu=0.05 \\
H_{0}: \mu_{C_{l}, \mathrm{MCS}}=\mu_{C_{l}, \mathrm{SDE}} \\
H_{1}: \mu_{C_{l}, \mathrm{MCS}} \neq \mu_{C_{l}, \mathrm{SDE}}\end{array}$} & \multicolumn{2}{|c|}{ computing time [s] } \\
\hline 50 & 0.00405 & 0.305 & & 0.230 & 0.243 \\
\hline 100 & 0.000703 & 0.441 & & 0.427 & 0.468 \\
\hline 150 & 0.00429 & 0.095 & 1.961 & 0.627 & 0.700 \\
\hline 200 & 0.00514 & 1.187 & & 0.819 & 0.990 \\
\hline
\end{tabular}


Case study 1: prognostic of electrolytic capacitors

\begin{tabular}{|c|c|c|c|c|c|}
\hline time $[\mathrm{h}]$ & $\mathrm{KL}\left(p_{\mathrm{MCS}}|| p_{\mathrm{SDE}}\right)$ & \multicolumn{2}{|c|}{$\begin{array}{l}\text { Hyp. test } @ \nu=0.05 \\
H_{0}: \mu_{C_{l}, \mathrm{MCS}}=\mu_{C_{l}, \mathrm{SDE}} \\
H_{1}: \mu_{C_{l}, \mathrm{MCS}} \neq \mu_{C_{l}, \mathrm{SDE}}\end{array}$} & \multicolumn{2}{|c|}{ computing time [s] } \\
\hline 50 & 0.00405 & 0.30 & & 0.230 & 0.243 \\
\hline 100 & 0.000703 & 0.44 & 1061 & 0.427 & 0.468 \\
\hline 150 & 0.00429 & 0.09 & 1.901 & 0.627 & 0.700 \\
\hline 200 & 0.00514 & 1.18 & & 0.819 & 0.990 \\
\hline
\end{tabular}


Case study 1: prognostic of electrolytic capacitors

Time-to-failure (TTF) prediction:

$$
\begin{gathered}
\mathbb{E}\left[\mathrm{T}_{\mathrm{F}}\right]=\frac{1}{\alpha} \ln \left(1-\frac{C_{l, t h}}{\beta}\right) \\
\mathrm{T}_{\mathrm{F}}^{(i)}=\frac{1}{\alpha} \ln \frac{C_{l, t h}-\beta}{\int_{0}^{\mathbb{E}\left[T_{F}\right]} \sigma e^{-\alpha s} \mathrm{~d} B_{s}-\beta} \quad \forall i=1, \ldots, N
\end{gathered}
$$




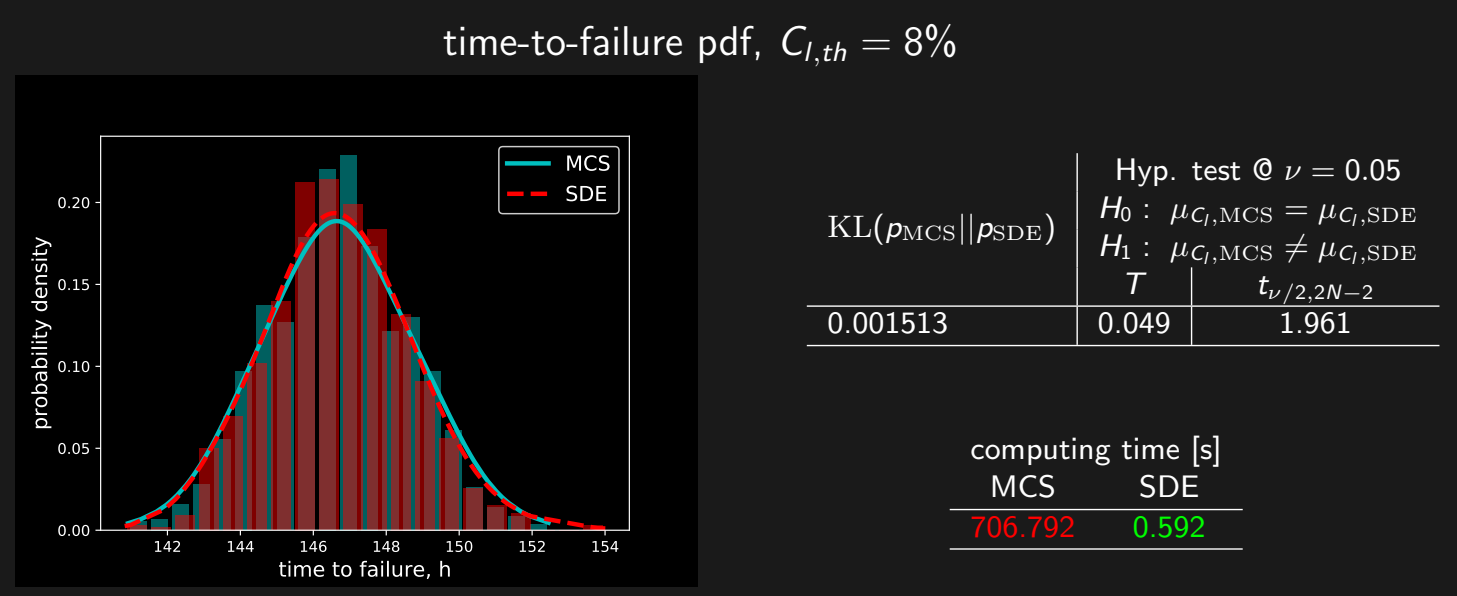


Case study 2: predicting the remaining time to discharge of Lithium-ion batteries using a simple state-of-charge (SOC) model $^{2}$ :

$R=$ internal resistance, $E=$ total energy delivered, $S=\mathrm{SOC}, \omega \cdot \sim \mathcal{N}\left(0, \sigma^{2}\right)$

$$
\begin{aligned}
& \dot{R}=\omega_{R} \\
& \dot{S}=-\frac{P}{E}+\omega_{S} \\
& \dot{E}=\omega_{E}
\end{aligned}
$$


Case study 2: remaining time to discharge of Lithium-ion batteries

We can directly sample from the pdfs of $R$ and $E$ at time $T$ :

$$
\begin{aligned}
& R_{T}^{(i)} \sim \mathcal{N}\left(R_{0}, \sigma_{R}^{2} T\right) \\
& E_{T}^{(i)} \sim \mathcal{N}\left(E_{0}, \sigma_{E}^{2} T\right)
\end{aligned}
$$


Case study 2: remaining time to discharge of Lithium-ion batteries

We can directly sample from the pdfs of $R$ and $E$ at time $T$ :

$$
\begin{aligned}
& R_{T}^{(i)} \sim \mathcal{N}\left(R_{0}, \sigma_{R}^{2} T\right) \\
& E_{T}^{(i)} \sim \mathcal{N}\left(E_{0}, \sigma_{E}^{2} T\right)
\end{aligned}
$$

the $i$-th SOC sample becomes:

$$
S_{T}^{(i)}=S_{0}-\frac{P}{E_{T}^{(i)}} t+\sigma_{S} \sqrt{T} z^{(i)}
$$

Current $i_{T}$ and voltage $V_{T}$ are then estimated from $R$ and $S$

$$
V_{T}=v_{o c, T}\left(S_{T}\right)-i_{T}\left(R_{T}, P\right) R_{T}+\omega_{V}
$$


Case study 2: remaining time to discharge of Lithium-ion batteries

$$
E, R, S \text { over time up to } T=200 \mathrm{~s}
$$

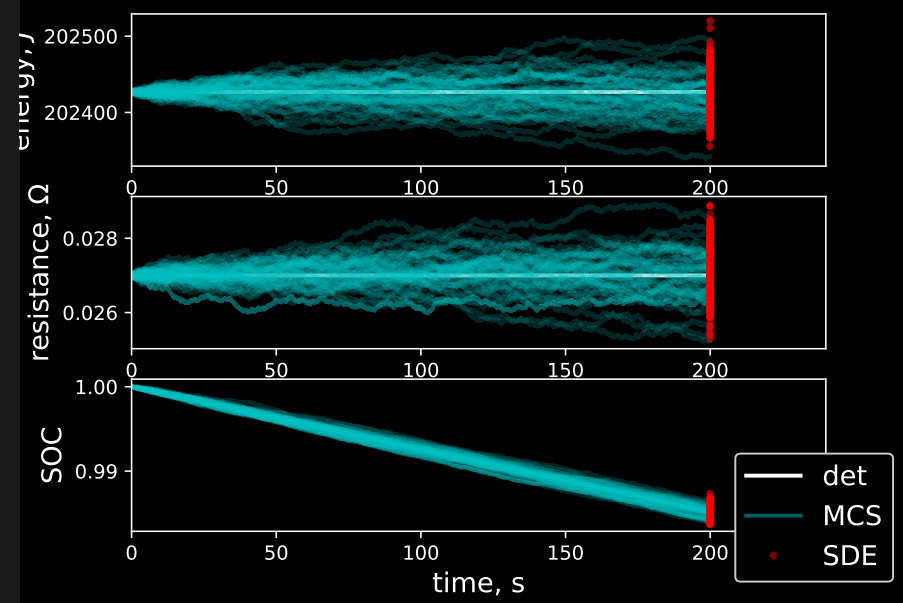


Case study 2: remaining time to discharge of Lithium-ion batteries

SOC pdf

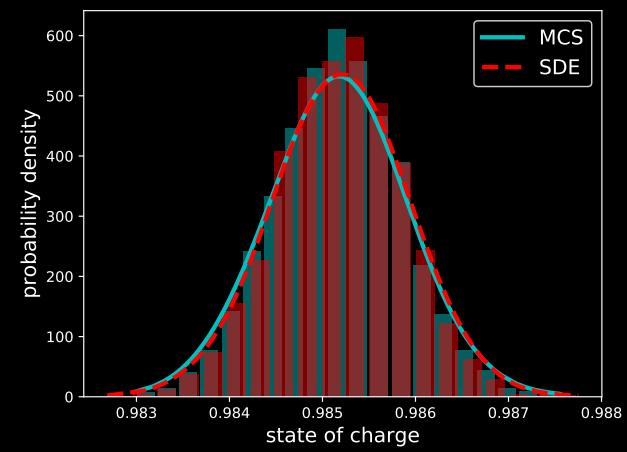

Voltage pdf

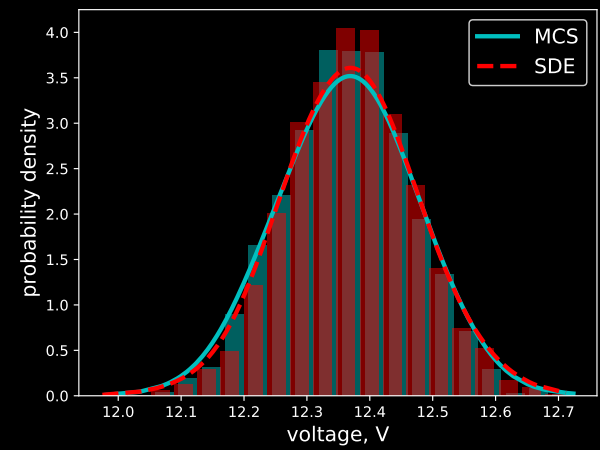


Case study 2: remaining time to discharge of Lithium-ion batteries

Comparing SOC distributions at $\mathrm{t}=200 \mathrm{~s}$

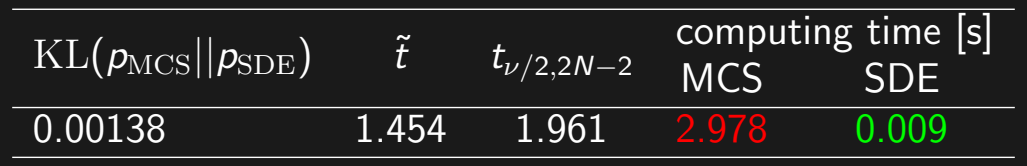


Case study 3: fatigue damage prognosis of cracked structure under constant amplitude fatigue loading, using Paris' law:

$$
\frac{\mathrm{d} a}{\mathrm{~d} n}=C^{\prime} a^{\gamma} e^{\omega}
$$


Case study 3: fatigue damage prognosis of cracked structure under constant amplitude fatigue loading, using Paris' law:

$$
\begin{gathered}
\frac{\mathrm{d} a}{\mathrm{~d} n}=C^{\prime} a^{\gamma} e^{\omega} \\
\frac{1}{C^{\prime}} \int_{a_{0}}^{a_{F}} \frac{1}{a^{\gamma}} \mathrm{d} a=\int_{0}^{n_{F}} e^{\omega_{s}} \mathrm{~d} s
\end{gathered}
$$


Case study 3: fatigue damage prognosis of cracked structure under constant amplitude fatigue loading, using Paris' law:

$$
\begin{gathered}
\frac{\mathrm{d} a}{\mathrm{~d} n}=C^{\prime} a^{\gamma} e^{\omega} \\
\frac{1}{C^{\prime}} \int_{a_{0}}^{a_{F}} \frac{1}{a^{\gamma}} \mathrm{d} a=\int_{0}^{n_{F}} e^{\omega_{s}} \mathrm{~d} s \\
\int_{0}^{\mathbb{E}\left[n_{F}\right]} e^{\omega_{s}} \mathrm{~d} s \approx \sum_{s=0}^{k-1} e^{\omega^{(i)}} \Delta n_{s} \quad \forall i=1, \ldots, N
\end{gathered}
$$


Case study 3: fatigue damage prognosis of cracked structure

FCG over time (up to $n=100000$ load cycles)

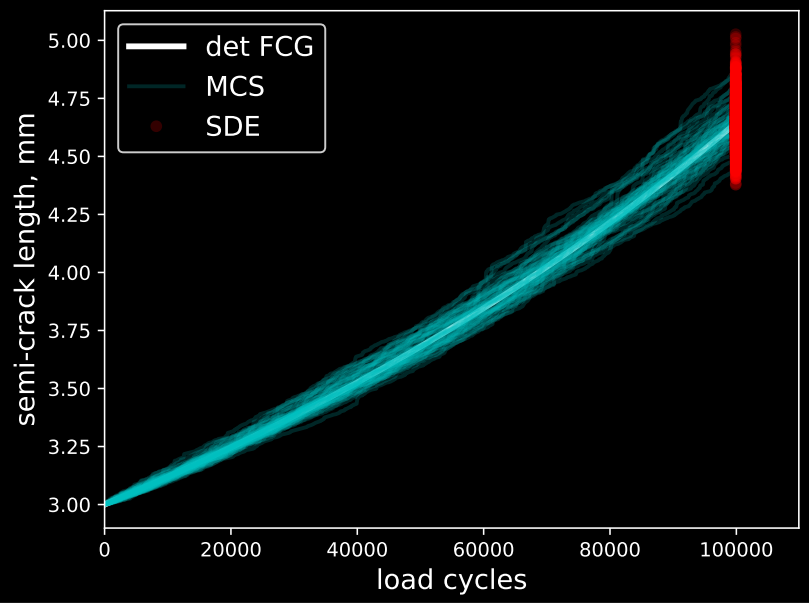




$$
\text { crack length pdf, } n=100000 \text { load cycles }
$$

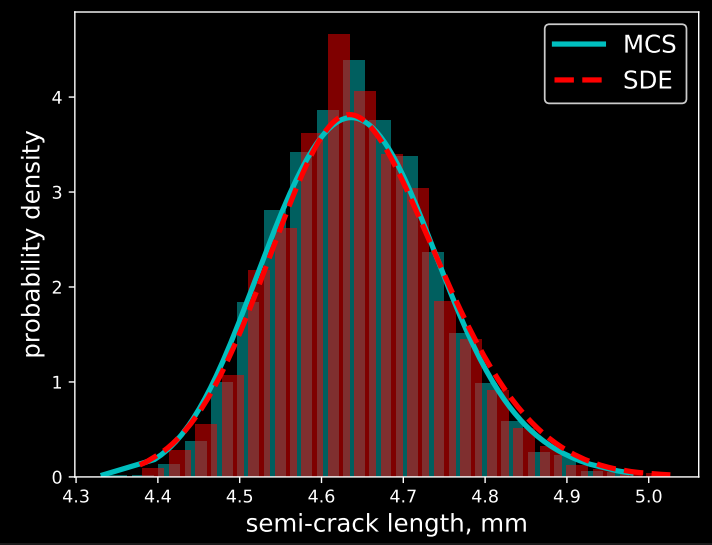

SDE method does not help in this case:

\begin{tabular}{lcc}
\hline $\mathrm{KL}\left(p_{\mathrm{MCS}} \| p_{\mathrm{SDE}}\right)$ & $\tilde{t}$ & $t_{\nu / 2,2 N-2}$ \\
\hline 0.00198 & 1.748 & 1.961
\end{tabular}

\begin{tabular}{|c|c|}
\hline comp & time \\
\hline MCS & SDE \\
\hline 0.180 & 0.205 \\
\hline
\end{tabular}


time-to-failure pdf, $a_{\text {th }}=6 \mathrm{~mm}$

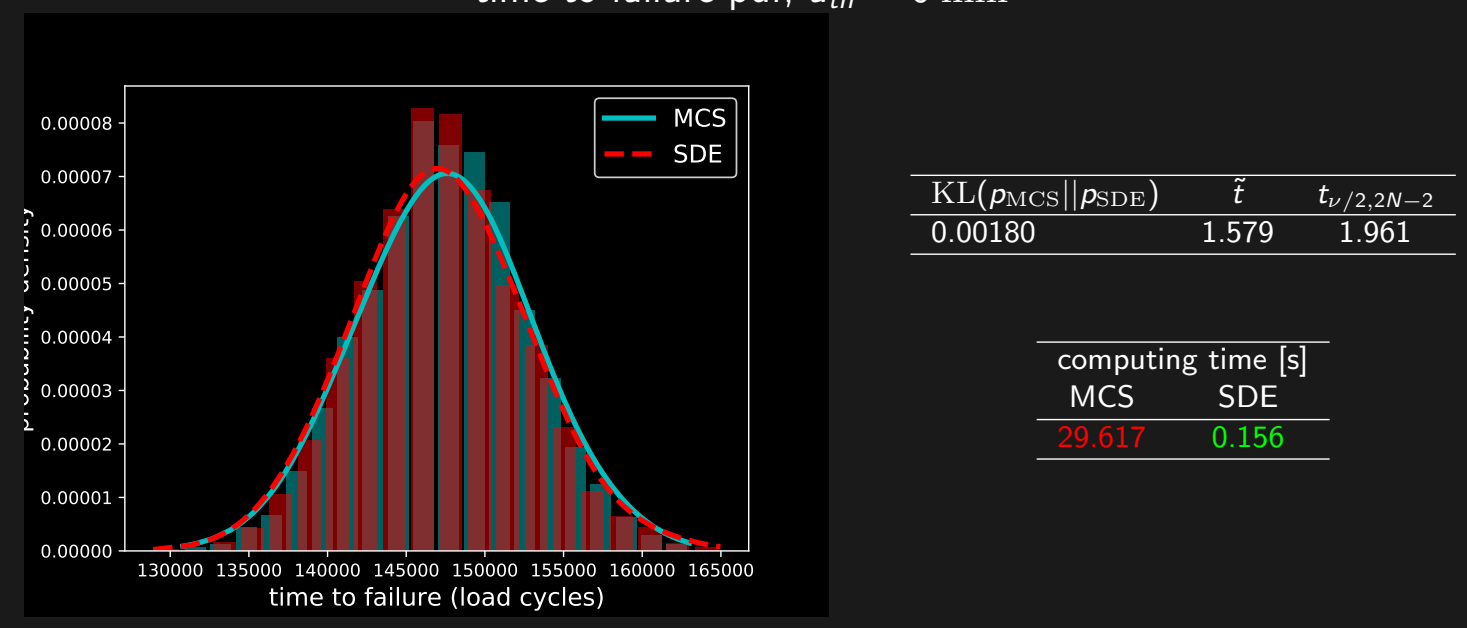




\section{Conclusions}

To summarize

- Fast MC approximation of prediction distributions using stochastic calculus

- Pro: pdfs of interest can be computed much faster

- Cons: limited to relatively simple models

- Cons: does not generalize easily, performance are model-dependent

\section{Future works}

- generalize to $x_{0} \sim p\left(X_{0}\right)$ and $\boldsymbol{\theta} \sim p(\boldsymbol{\theta})$ before deployment.

- extension to vector SDEs and other model classes, whenever possible

- sensitivity analysis: number of samples, number of prediction steps, etc.

A fast Monte Carlo method for model-based prognostic based on stochastic calculus Matteo Corbetta, Chetan Kulkarni - SGT, NASA Ames Research Center matteo.corbetta@nasa.gov 